\title{
Quantum probes for the cutoff frequency of Ohmic environments
}

\author{
Claudia Benedetti, ${ }^{1}$ Fahimeh Salari Sehdaran, ${ }^{2}$ Mohammad H. Zandi, ${ }^{2}$ and Matteo G. A. Paris ${ }^{1}$ \\ ${ }^{1}$ Quantum Technology Lab, Physics Department, Università degli Studi di Milano, Milano, Italy \\ ${ }^{2}$ Faculty of Physics, Shahid Bahonar University of Kerman, Kerman, Iran
}

(Received 6 October 2017; published 24 January 2018)

\begin{abstract}
Quantum probing consists of suitably exploiting a simple, small, and controllable quantum system to characterize a larger and more complex system. Here, we address the estimation of the cutoff frequency of the Ohmic spectral density of a harmonic reservoir by quantum probes. To this aim, we address the use of single-qubit and two-qubit systems and different kinds of coupling with the bath of oscillators. We assess the estimation precision by the quantum Fisher information of the sole quantum probe as well as the corresponding quantum signal-to-noise ratio. We prove that, for most of the values of the Ohmicity parameter, a simple probe such as a single qubit is already optimal for the precise estimation of the cutoff frequency. Indeed for those values, upon considering a two-qubit probe either in a Bell or in separable state, we do not find improvement to the estimation precision. However, we also showed that there exist few conditions where employing two qubits in a Bell state interacting with a common bath is more suitable for precisely estimating the cutoff frequency.
\end{abstract}

DOI: 10.1103/PhysRevA.97.012126

\section{INTRODUCTION}

Complex quantum systems with many degrees of freedom are often difficult to access and, in turn, to characterize. A possible strategy to overcome this difficulty is that of monitoring only a small portion of the system and exploiting an indirect measurement scheme to estimate the value of the parameters of interest. An effective way to implement this paradigm is by means of quantum probes. A quantum probe is a simple and controllable quantum system that interacts with a larger reservoir (also refereed to as an environment or bath) and becomes entangled with it. Due to quantum correlations the probe becomes extremely sensitive to the perturbations induced by the environment, and upon performing a measurement on the quantum probes one may effectively infer the properties of the environment [1-6], i.e., extract information on the parameter of interest. The outcomes of the measurement performed on the probe are then used to build an estimator for the unknown parameter, whose precision can be assessed using the tools of quantum estimation theory (QET) [7]. Indeed, QET has already proven useful in different contexts, ranging from the estimation of the spectral properties of the environment [8-11] to quantum channel parameters [12-15], quantum correlations [16-19], optical phases [20-24], quantum thermometry $[25,26]$, and the coupling constants of different kinds of interactions [27-31]. In particular, the quantum Fisher information (QFI) is the quantity that allows us to evaluate the ultimate precision of any estimation procedure as ruled by quantum mechanics through the quantum CramérRao bound (CRB). The larger the QFI, the more accurate is the estimation strategy.

A relevant quantity to characterize complex environments is the so-called spectral density, which is the Fourier transform of its autocorrelation function and, in turn, determines how and how fast quantum probes are going to decohere. In devices of interest for quantum technology this determines the available coherence time for communication and computation, and thus a precise characterization of the spectral density is a crucial step to design engineered reservoirs. Thermal noise shows a flat spectrum, while in structured reservoirs as those encountered working with Josephson junctions [32] or photonic crystals [33], different spectra may be observed. In this framework, a crucial parameter characterizing a complex environment is its cutoff frequency, which is linked to the environment correlation time as $\tau_{c}=1 / \omega_{c}$ and represents the frequency above which the spectral density starts to fall off.

In particular, in this work we consider an exponential cutoff function and address the estimation of the cutoff frequency for the Ohmic family of spectral densities characterizing a bosonic reservoir. In order to pursue this task, we consider single- and two-qubit systems interacting with their environment and use them as quantum probes. This means optimizing the initial preparation of the probe and performing a measurement on the system to extract information about the spectral cutoff frequency. Due to the interaction with the environment, the quantum probes will be generally subjected to decoherence (dephasing) and dissipation phenomena. The time scales of these processes depend on the physical context considered. Usually, the dissipation time scale is much longer than the decoherence one, such that the dynamics of many systems of interest may be described as pure dephasing [34,35], and this is indeed the case considered here.

We compare the behavior of the quantum Fisher information and the signal-to-noise ratio (SNR) for different values of the Ohmic parameter, moving from sub-Ohmic to super-Ohmic regimes. We first study the case of a single qubit used as a probe; then we extend our analysis to the two-qubit scenario, in both independent and common environments. In this way, we try to understand whether multiple (and entangled) probes may improve the estimation procedure. We compare the maximized QFI at the optimal interaction time and prove that, for most of the Ohmicity parameter values, a single quantum probe is already sufficient to achieve optimal estimation of the parameter. 
The paper is organized as follows: In Sec. II, we introduce the physical model, whereas in Sec. III we briefly summarize the tools of local estimation theory. In Sec. IV, we present our results on the precision achievable by quantum probes in the estimation of the cutoff frequency of the spectral density. Section V closes the paper with some concluding remarks.

\section{THE PHYSICAL MODEL}

We consider a pure dephasing model consisting of one or two qubits which interact with a bosonic reservoir at zero temperature, characterized by an Ohmic spectral density. This model allows for an exact analytic solution $[1,36]$ and many of its features have already been analyzed [37-41]. Here we change the point of view with respect previous studies; i.e., we use the qubits as quantum probes for a spectral parameter of the system-reservoir couplings, rather than looking for the decoherence effects on the qubits assuming the knowledge of the reservoir.

\section{A. Single qubit}

We first focus on a single-qubit probe, characterized by energy spacing $\omega_{0}$, coupled with all the modes of a bath of harmonic oscillators (hereafter we set $\hbar=1$ and we scale all frequencies with $\omega_{0}$ ). The global dimensionless Hamiltonian $\mathcal{H}=\mathcal{H}_{S}+\mathcal{H}_{B}+\mathcal{H}_{I}$ is given by

$$
\mathcal{H}=\frac{1}{2} \sigma_{z}+\sum_{k} \omega_{k} b_{k}^{\dagger} b_{k}+\sum_{k} \sigma_{z}\left(g_{k} b_{k}^{\dagger}+g_{k}^{*} b_{k}\right),
$$

where $\sigma_{z}$ is the Pauli operator of the qubit, $b_{k}^{\dagger}\left(b_{k}\right)$ denotes bosonic creation (annihilation) operator for mode $k$, satisfying the commutation relation $\left[b_{k}, b_{k^{\prime}}^{\dagger}\right]=\delta_{k k^{\prime}}, \omega_{k}$ is the frequency of the $k$ th mode, and $g_{k}$ is the corresponding coupling constant with the qubit. Both $\omega_{k}$ and $g_{k}$ are expressed in units of $\omega_{0}$ and are thus dimensionless.

The couplings $g_{k}$ can be distributed according to different spectral distributions, which lead to different dynamical properties for the qubit. Following [1,36], we can calculate the reduced dynamics of the qubit in the interaction picture. We suppose that the bath is initially in a thermal state at zero temperature. If we move to a continuum limit $\omega_{k} \rightarrow$ $\omega(k)$ and $\sum_{k} \rightarrow \int d \omega f(\omega)$, with $f(\omega)$ the density of modes, we can introduce the spectral density $J(\omega)=4 f(\omega)|g(\omega)|^{2}$. Assuming that the couplings $g(\omega)$ are nearly constant in $\omega$, $J(\omega)$ becomes the spectral density of the bath's modes. Here we consider a reservoir with a spectral density belonging to the Ohmic class:

$$
J\left(\omega, \omega_{c}\right)=\frac{\omega^{s}}{\omega_{c}^{s-1}} e^{-\frac{\omega}{\omega_{c}}},
$$

parametrized by a real positive number $s$, which moves the spectrum from sub-Ohmic $(s<1)$ to Ohmic $(s=1)$ and super-Ohmic $(s>1)$ regimes. Common values of $s$ are $0.5,1$, and 3 , used to describe quantum Brownian motion, conductive electrons in metals, phonon baths, $1 / f^{\alpha}$ noise in solids and in superconducting qubits, and the interaction between a charged particle and its own electromagnetic field [42-45]. $\omega_{c}$ is the cutoff frequency, i.e., the parameter we want to estimate using quantum probes. Once the spectral density is fixed, the qubit dynamics can be easily calculated through the single-qubit quantum map $\Phi(t)$ :

$$
\rho(t)=\Phi(t) \circ \rho(0)
$$

where

$$
\Phi(t)=\left(\begin{array}{cc}
1 & e^{-\Gamma\left(t, \omega_{c}\right)} \\
e^{-\Gamma\left(t, \omega_{c}\right)} & 1
\end{array}\right),
$$

where $\rho(0)$ is the initial state of the qubit, $t$ is the dimensionless time, and $\circ$ is the element-wise Hadamard product [37]. The decoherence factor $\Gamma\left(t, \omega_{c}\right)$ depends upon the spectral density of the bath and takes the form

$$
\Gamma\left(t, \omega_{c}\right)=\int_{0}^{\infty} \frac{1-\cos (\omega t)}{\omega^{2}} J\left(\omega, \omega_{c}\right) d \omega .
$$

The explicit expression of Eq. (5) depends on the Ohmicity parameter $s$ :

$$
\Gamma\left(t, \omega_{c}\right)=\left\{\begin{array}{cc}
\frac{1}{2} \ln \left(1+\left(\omega_{c} t\right)^{2}\right), & s=1 \\
\left(1-\frac{\cos \left[(s-1) \arctan \left(\omega_{c} t\right)\right]}{\left[1+\left(\omega_{c} t\right)^{2}\right]^{\frac{s-1}{2}}}\right) \bar{\Gamma}[s-1], & s \neq 1,
\end{array}\right.
$$

where $\bar{\Gamma}[x]=\int_{0}^{\infty} t^{x-1} e^{-t} d t$.

\section{B. Two qubits}

We now analyze the case of two noninteracting qubits coupled with the bosonic reservoir. Two different scenarios arise: either the two qubits are coupled to two independent local reservoirs, or they are embedded in the same bath.

\section{Two qubits in independent environments}

In the case of two noninteracting qubits coupled to independent but identical environments, the global Hamiltonian is

$$
\mathcal{H}=\mathcal{H}^{(1)}+\mathcal{H}^{(2)},
$$

where the dimensionless single-qubit Hamiltonian $\mathcal{H}^{(j)}, j=$ 1,2 , is given by

$$
\mathcal{H}^{(j)}=\frac{1}{2} \sigma_{z}^{(j)}+\sum_{k} \omega_{k} b_{k}^{\dagger(j)} b_{k}^{(j)}+\sum_{k} \sigma_{z}^{(j)}\left(g_{k} b_{k}^{\dagger(j)}+g_{k}^{*} b_{k}^{(j)}\right)
$$

and we assume that the qubits are coupled to their respective baths with the same strengths $g_{k}^{(1)}=g_{k}^{(2)} \forall k$. The two-qubit density matrix has the form

$$
\rho_{I}(t)=\Phi_{I}(t) \circ \rho(0),
$$

where the two-qubit map is the tensor product of the singlequbit channel (4),

$$
\Phi_{I}(t)=\Phi(t) \otimes \Phi(t)
$$

and $\rho(0)$ is the initial state of the two qubits.

\section{Two qubits in a common environment}

We now assume that the two qubits are now coupled to the same reservoir. The total Hamiltonian is

$$
\mathcal{H}=\frac{1}{2} \sum_{j=1}^{2} \sigma_{z}^{(j)}+\sum_{k} \omega_{k} b_{k}^{\dagger} b_{k}+\sum_{j=1}^{2} \sum_{k} \sigma_{z}^{(j)}\left(g_{k} b_{k}^{\dagger}+g_{k}^{*} b_{k}\right),
$$


where again we assume that the two qubits have the same couplings $g_{k}$ to the environment. Moving to the interaction picture and calculating the reduced dynamics of the two qubits, one obtains

$$
\rho_{c}(t)=\Phi_{c}(t) \circ \rho(0),
$$

where the map is

$$
\Phi_{c}(t)=\left(\begin{array}{cccc}
1 & e^{-\Gamma\left(t, \omega_{c}\right)} & e^{-\Gamma\left(t, \omega_{c}\right)} & e^{-4 \Gamma\left(t, \omega_{c}\right)} \\
e^{-\Gamma\left(t, \omega_{c}\right)} & 1 & 1 & e^{-\Gamma\left(t, \omega_{c}\right)} \\
e^{-\Gamma\left(t, \omega_{c}\right)} & 1 & 1 & e^{-\Gamma\left(t, \omega_{c}\right)} \\
e^{-4 \Gamma\left(t, \omega_{c}\right)} & e^{-\Gamma\left(t, \omega_{c}\right)} & e^{-\Gamma\left(t, \omega_{c}\right)} & 1
\end{array}\right)
$$

and $\Gamma\left(t, \omega_{c}\right)$ is defined in Eq. (6).

\section{LOCAL QUANTUM ESTIMATION THEORY}

Consider a family of quantum states $\rho_{\omega_{c}}$ depending on an unknown parameter $\omega_{c}$. In order to infer the value of the parameter we perform a large number of repeated measurements on the system and then process the outcomes to build an estimator $\hat{\omega}_{c}$ for the parameter. This procedure will inevitably associate an error to the estimator, which can be quantified through its variance $\sigma^{2}$. Local quantum estimation theory (LQET) tells us which estimation strategies lead to precise estimators, comparing the Fisher information (FI) of a certain measurement with the QFI. Indeed, there is a bound to the precision of any unbiased estimator, given by the Cramér-Rao inequality,

$$
\sigma^{2}\left(\hat{\omega}_{c}\right) \geqslant \frac{1}{M F\left(\omega_{c}\right)}
$$

where $M$ is the number of repeated measurements and $F\left(\omega_{c}\right)$ is the Fisher information associated to a certain measurement whose outcomes $\{x\}$ are distributed according to the conditional probability $p\left(x \mid \omega_{c}\right)$ :

$$
F\left(\omega_{c}\right)=\int d x \frac{1}{p\left(x \mid \omega_{c}\right)}\left(\frac{\partial p\left(x \mid \omega_{c}\right)}{\partial \omega_{c}}\right)^{2} .
$$

The CRB (14) can further be bounded by the quantum CramérRao bound (QCRB)

$$
\sigma^{2}\left(\hat{\omega}_{c}\right) \geqslant \frac{1}{M H\left(\omega_{c}\right)},
$$

where we introduced the QFI $H\left(\omega_{c}\right)$, obtained by maximizing the FI over all possible measurements [7].

The explicit expression of the QFI can be found after diagonalizing the density matrix of the system of interest, $\rho_{\omega_{c}}=\sum_{n} \rho_{n}\left|\phi_{n}\right\rangle\left\langle\phi_{n}\right|:$

$$
H\left(\omega_{c}\right)=\sum_{n} \frac{\left(\partial_{\omega_{c}} \rho_{n}\right)^{2}}{\rho_{n}}+2 \sum_{n \neq m} \frac{\left(\rho_{n}-\rho_{m}\right)^{2}}{\rho_{n}+\rho_{m}}\left|\left\langle\phi_{m} \mid \partial_{\omega_{c}} \phi_{n}\right\rangle\right|^{2},
$$

where $\partial_{\omega_{c}}$ is the derivative with respect the parameter $\omega_{c}$. The first term in Eq. (17) is the classical FI of the distribution $\left\{\rho_{n}\right\}$, while the second term is quantum in nature and vanishes when the eigenvectors of $\rho_{\omega_{c}}$ do not depend on the parameter $\omega_{c}$. Another figure of merit that can be addressed in order to evaluate the precision of an estimator is the SNR $r\left(\omega_{c}\right)=$ $\frac{\omega_{c}^{2}}{\sigma^{2}\left(\omega_{c}\right)}$. This quantity is always bounded from above by the quantum signal-to-noise ratio (QSNR), defined as

$$
R\left(\omega_{c}\right)=\omega_{c}^{2} H\left(\omega_{c}\right) .
$$

A large value of the QSNR thus means that the parameter can be estimated efficiently, with a small error.

\section{CUTOFF FREQUENCY ESTIMATION BY QUANTUM PROBES}

In this section we report our results about the estimability of the cutoff frequency of the spectral density $J(\omega)$ belonging to the Ohmic family. This is achieved by analyzing the behavior of the QFI and the QSNR for fixed values of the Ohmicity parameter $s$. In the case of a single qubit we are able to find the optimal preparation state, which maximizes the QFI, and the optimal measure, such that its FI equals the QFI, i.e., $F\left(\omega_{c}\right)=$ $H\left(\omega_{c}\right)$. In the case of two qubits, we compare the QFI for different initial states, i.e., product and entangled states, in both common and independent environments (see Table I). Our aim is to understand whether quantum correlations can improve the estimation precision or if a single qubit is already sufficient for efficient estimation. Indeed we bring evidence that a simple quantum probe like a single qubit is enough to efficiently estimate the cutoff frequency of an Ohmic spectral density in a dephasing dynamics.

\section{A. Single qubit}

In this section we analyze the estimability of the cutoff frequency of the spectral density belonging to the Ohmic family (2) using a single qubit as a quantum probe. We initially prepare the qubit in a pure state depending upon the parameter $\theta$ :

$$
\left|\psi_{0}\right\rangle=\cos \left(\frac{\theta}{2}\right)|0\rangle+\sin \left(\frac{\theta}{2}\right)|1\rangle .
$$

The QFI can be analytically computed according to Eq. (17) after diagonalizing the density matrix for the qubit $\rho_{0}=$ $\left|\psi_{0}\right\rangle\left\langle\psi_{0}\right|$ :

$$
H\left(t, \omega_{c}\right)=\frac{\sin ^{2} \theta\left[\partial_{\omega_{c}} \Gamma\left(t, \omega_{c}\right)\right]^{2}}{e^{2 \Gamma\left(t, \omega_{c}\right)}-1},
$$

which is maximized for $\theta=\frac{\pi}{2}$ such that the optimal initial state preparation is $|+\rangle=\frac{1}{\sqrt{2}}(|0\rangle+|1\rangle)$, independent on the value of $\omega_{c}$ and the interaction time.

We recognize that the QFI coincides with the FI of population measurement of the qubit diagonalized density matrix [9]:

$$
H\left(t, \omega_{c}\right)=\frac{\left[\partial_{\omega_{c}} \Gamma\left(t, \omega_{c}\right)\right]^{2}}{e^{2 \Gamma\left(t, \omega_{c}\right)}-1} .
$$

By substituting the explicit form of $\Gamma\left(t, \omega_{c}\right)$ [Eq. (6)] into the above equation, one gets the analytical expression of the decoherence coefficient for fixed values of $s$.

In order to optimize the inference procedure, we look for the interaction time that maximizes the QFI as a function of $\omega_{c}$ and for a fixed value of $s$. The maximization of the QFI over time has been performed numerically. The optimal 
TABLE I. Summary of results for two-qubit quantum probes. We compare four different estimation schemes for the cutoff frequency of the spectral density $J(\omega)$ in Eq. (2) using two qubits as quantum probes: (a) qubits prepared in a separable state interacting with independent and identical reservoirs, (b) qubits prepared in a Bell state interacting with independent and identical reservoirs, (c) qubits prepared in a separable state coupled to a common bath, and (d) qubits prepared in a Bell state coupled to a common bath. We also report the expressions for their respective QFI as a function of the decoherence factor $\Gamma$.

(a)

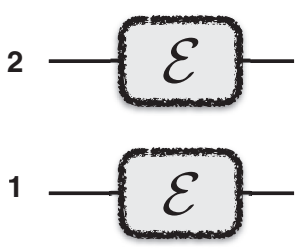

$H_{I}^{P}=2 \frac{\left[\partial_{\omega_{c}} \Gamma\left(t, \omega_{c}\right)\right]^{2}}{e^{2 \Gamma\left(t, \omega_{c}\right)}-1}$ (b)

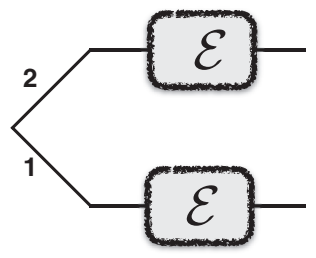

$H_{I}^{B}=4 \frac{\left[\partial_{\omega_{c}} \Gamma\left(t, \omega_{c}\right)\right]^{2}}{e^{4 \Gamma\left(t, \omega_{c}\right)}-1}$ (c)

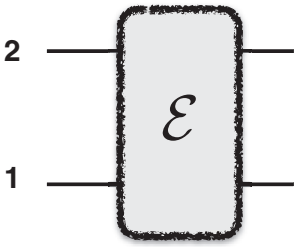

$H_{c}^{P}=\frac{8\left\{1+e^{4 \Gamma\left(t, \omega_{c}\right)}\left[1+\sinh \left(2 \Gamma\left(t, \omega_{c}\right)\right)\right]\right\}\left[\partial_{\omega_{c}} \Gamma\left(t, \omega_{c}\right)\right]^{2}}{3 e^{8 \Gamma\left(t, \omega_{c}\right)}-2 e^{4 \Gamma\left(t, \omega_{c}\right)}-1}$ (d)

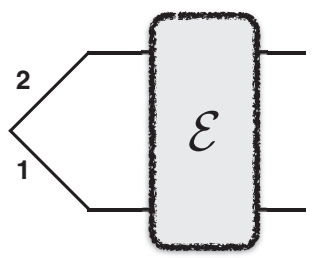

$H_{c}^{B}=\frac{16\left[\partial_{\omega_{c}} \Gamma\left(t, \omega_{c}\right)\right]^{2}}{e^{8 \Gamma\left(t, \omega_{c}\right)}-1}$ time $t^{\mathrm{opt}}\left(s, \omega_{c}\right)$, where the quantum Fisher information has a maximum for every value of $s$, is inversely proportional to the cutoff frequency while the quantum Fisher information calculated at the optimal time is inversely proportional to the square of $\omega_{c}$ :

$$
t^{\mathrm{opt}}\left(s, \omega_{c}\right)=\frac{G(s)}{\omega_{c}}, \quad H\left(t^{\mathrm{opt}}, s, \omega_{c}\right)=\frac{R(s)}{\omega_{c}^{2}},
$$

as shown in Fig. 1. The quantity $G(s)$ does not depend on the value of the parameter to be estimated, $\omega_{c}$, but only on the Ohmicity $s$. When we substitute the optimal time into the expression for $H$, we obtain that the optimized QFI scales with the inverse of $\omega_{c}^{2}$. This means that the QSNR $R\left(t^{\mathrm{opt}}, s, \omega_{c}\right)=\omega_{c}^{2} H\left(t^{\mathrm{opt}}, s, \omega_{c}\right)$ is independent of the value of $\omega_{c}$ since it depends only on the parameter $s$. The QSNR has the

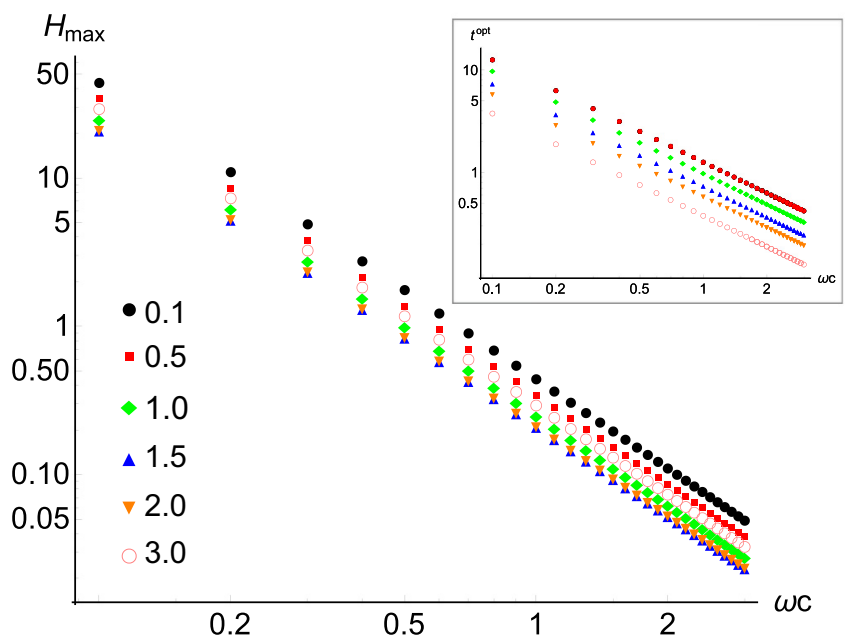

FIG. 1. Quantum Fisher information $H_{\max }$ and optimal time $t^{\text {opt }}$ (inset) as a function of $\omega_{c}$ for different values of the parameter $s$ (in the legend), in the single-qubit case. expression

$$
R(s)= \begin{cases}\frac{G^{2}(s)}{\left(1+G^{2}(s)\right)^{2}}, & s=1 \\ \frac{\operatorname{coth}\left[\left(1-\frac{\cos [(s-1) \arctan G(s)]}{\left(1+G^{2}(s)\right)^{\frac{s-1}{2}}}\right) \bar{\Gamma}[s-1]\right]-1}{2 \frac{\left(1+G^{2}(s)\right)^{s}}{G^{2}(s) \bar{\Gamma}[s]^{2}} \csc ^{2}[s \arctan G(s)]}, & s \neq 1,\end{cases}
$$

where $G(s)$ is the proportionality constant of the optimal time (22). Both $G(s)$ and $R(s)$ are reported in Fig. 2, which shows us that $R(s)$ has a nonmonotone behavior in $s$, with a global minimum.

The fact that $R(s)$ is independent on the value of $\omega_{c}$ means that using a single qubit as a quantum probe allows a uniform estimation of the cutoff frequency. For small values of $s$ the QSNR decreases, then it reaches a minimum, after which it starts increasing until it saturates to a constant value for large values of $s$.

\section{B. Two qubits}

We now focus on the situation where two qubits are used as quantum probes, in order to understand whether multiple

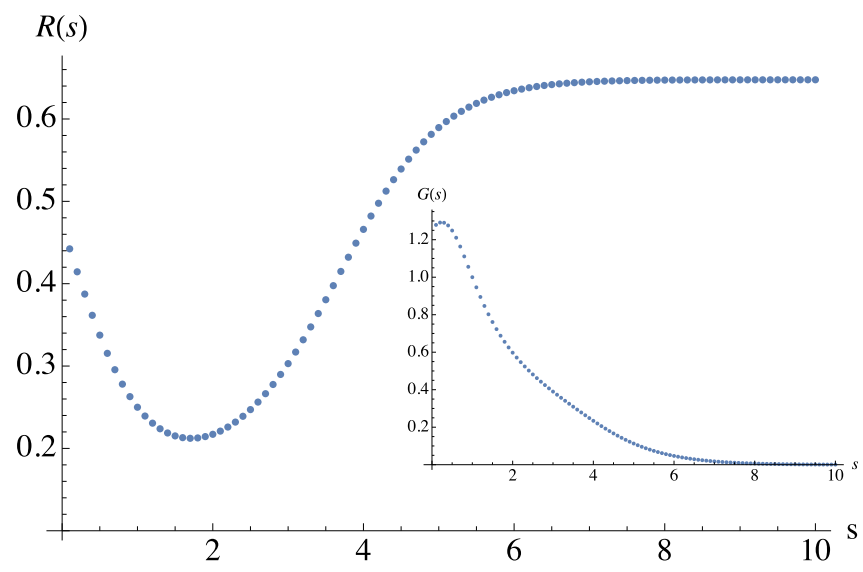

FIG. 2. Dependency of the QSNR $R$ on the parameter $s$ for the single-qubit case. In the inset we report the behavior of the coefficient $G$ as a function of $s$. 


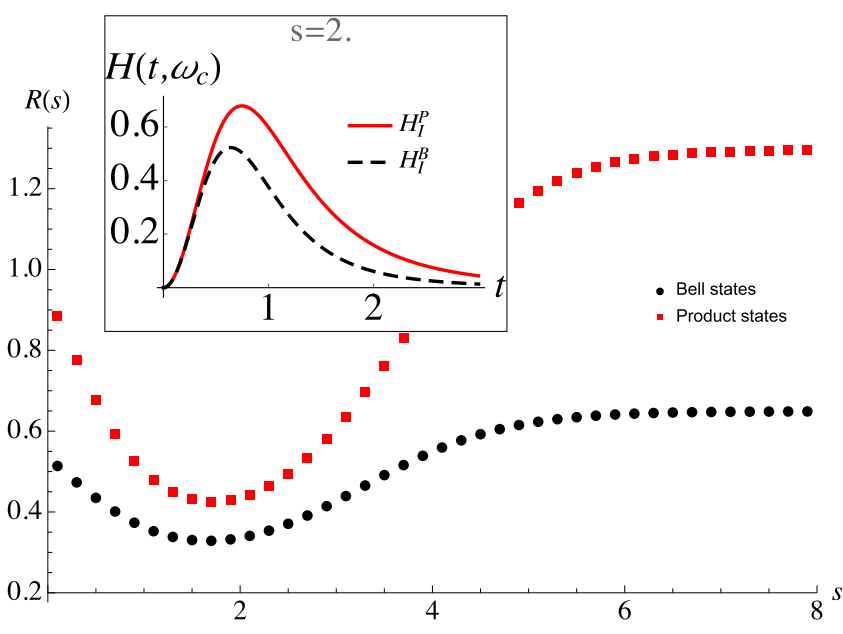

FIG. 3. Dependency of the QSNR $R$ on the parameter $s$, in the case of two qubits interacting with identical independent baths. In the inset we compare the QFI for product (solid red line) and Bell (dashed black line) states as a function of time for $\omega_{c}=0.8$.

quantum probes perform better than a single qubit. The maximization over a generic initial state of the qubits is not trivial in this case. For this reason we focus on two different state preparations, i.e., the four product states $| \pm \pm\rangle,| \pm \mp\rangle$ and the four Bell states $\left|\phi^{ \pm}\right\rangle$and $\left|\psi^{ \pm}\right\rangle$, where $\left|\phi^{ \pm}\right\rangle=\frac{1}{\sqrt{2}}(|00\rangle \pm|11\rangle)$ and $\left|\psi^{ \pm}\right\rangle=\frac{1}{\sqrt{2}}(|01\rangle \pm|10\rangle)$. Moreover, different scenarios are considered: we start with the case where two qubits interact with independent local reservoirs and then we analyze the case of two qubits in a common bath.

In the case of two qubits in independent environments [Tables I(a) and I(b)], we find that all four product states lead to the same QFI, which is twice the single-qubit QFI $H\left(t, \omega_{c}\right)$ of Eq. (20), thus confirming the additivity of the quantum Fisher information:

$$
H_{I}^{P}\left(t, \omega_{c}\right)=2 H\left(t, \omega_{c}\right)
$$

Also in the case where the two qubits are initially entangled, the QFI is the same for all four Bell states, and it reads

$$
H_{I}^{B}\left(t, \omega_{c}\right)=4 \frac{\left[\partial_{\omega_{c}} \Gamma\left(t, \omega_{c}\right)\right]^{2}}{e^{4 \Gamma\left(t, \omega_{c}\right)}-1} .
$$

After maximizing both $H_{I}^{P}\left(t, \omega_{c}\right)$ and $H_{I}^{B}\left(t, \omega_{c}\right)$ over time, we find the same dependency as in the case of the single qubit: the optimal time is inversely proportional to the cutoff frequency and the maximized QFI scales as $\omega_{c}^{-2}$, as reported in Eq. (22). The optimal time for product states is always larger than $t^{\text {opt }}$ for Bell states but if we fix a target precision much smaller than the QCRB, product and Bell states can achieve it at the same time (shown in the inset of Fig. 3), while an intermediate precision will be obtained faster by employing product states. Indeed, for small times $t \ll 1$, we can expand in series to third order the QFI in both cases:

$$
\begin{aligned}
& H_{I}^{P}\left(t, \omega_{c}\right)=2 \bar{\Gamma}[1+s] t^{2}-\frac{\omega_{c}^{2}}{2}\left(2 \bar{\Gamma}[1+s]^{2}+\bar{\Gamma}[3+s]\right) t^{4}, \\
& H_{I}^{B}\left(t, \omega_{c}\right)=2 \bar{\Gamma}[1+s] t^{2}-\frac{\omega_{c}^{2}}{2}\left(4 \bar{\Gamma}[1+s]^{2}+\bar{\Gamma}[3+s]\right) t^{4},
\end{aligned}
$$

and we see that up to second order the two expansions coincide.

The QFI at its optimal time is always higher for initial product states than for Bell states for a fixed value of $\omega_{c}$, and since it is proportional to $\omega_{c}^{-2}$ it follows that the QSNR is constant and depends only on $s$. Since we are interested in the maximum precision allowed by quantum mechanics, we consider as a figure of merit to the goodness of the inferring procedure the QSNR evaluated at the optimal time. In Fig. 3 we compare the behavior of $R\left(t^{\mathrm{opt}}, s, \omega_{c}\right)$ for initial product and Bell states.

As it is apparent from the plot, the quantum correlations of Bell states do not help in estimating the unknown parameter. Indeed product states allow us to obtain a larger QSNR for a fixed values of the Ohmicity $s$, i.e., a more precise inference of $\omega_{c}$.

We now consider the case where the two qubits interact with the same environment, as shown in Tables $\mathrm{I}(\mathrm{c})$ and $\mathrm{I}(\mathrm{d})$. All four product states will give the same QFI:

$$
\begin{aligned}
H_{c}^{P}\left(t, \omega_{c}\right)= & {\left[\partial_{\omega_{c}} \Gamma\left(t, \omega_{c}\right)\right]^{2} } \\
& \times \frac{8\left\{1+e^{4 \Gamma\left(t, \omega_{c}\right)}\left[1+\sinh \left(2 \Gamma\left(t, \omega_{c}\right)\right)\right]\right\}}{3 e^{8 \Gamma\left(t, \omega_{c}\right)}-2 e^{4 \Gamma\left(t, \omega_{c}\right)}-1},
\end{aligned}
$$

while for Bell states, only the $\left|\psi^{ \pm}\right\rangle$give a significant contribution, with a QFI equal to

$$
H_{c}^{B}\left(t, \omega_{c}\right)=\frac{16\left[\partial_{\omega_{c}} \Gamma\left(t, \omega_{c}\right)\right]^{2}}{e^{8 \Gamma\left(t, \omega_{c}\right)}-1} .
$$

As before, we are interested in the optimized QFI: we find that it is inversely proportional to $\omega_{c}^{2}$, such that the QSNR is constant for a fixed value of $s$. Our previous result holds true for most of the values of the parameter $s$; i.e., product states in independent baths yield the higher value of the QSNR compared to the other scenarios. However, there exists a range of values of the Ohmicity parameter for which the $R(s)$ is larger if we employ the Bell states in a common-bath scheme. This is shown in Fig. 4, where we compare the behavior of the QFI for two different values of $s$ as a function of time in the four estimation schemes considered in this paper (top plots) and the QSNR as a function of $s$ (bottom plot). In particular, we emphasize the fact that there exist values of $s$, such as $s=2$ in our example, where the estimation of the cutoff frequency is improved if we employ a common-bath scheme with two qubits in a Bell state. Indeed, we see that $H_{c}^{B}\left(t^{\mathrm{opt}}, \omega_{c}\right)$ is larger than $H_{I}^{P}\left(t^{\mathrm{opt}}, \omega_{c}\right)$. It is also worth noticing that in this case the optimal time for $H_{c}^{B}$ is shorter than that of independent probes.

Since employing two noninteracting qubits that are coupled to independent identical reservoirs initialized in a separable state is the same as repeating twice the single-qubit procedure described in Sec. II, it follows that using a single qubit as a probe is sufficient to optimally estimate the cutoff frequency of an Ohmic spectral density for most values of $s$. This is due to the fact that, for those values of $s$, using multiple qubits in a Bell state, in common or independent reservoirs, does not lead to improvements in the estimation procedure. Common 


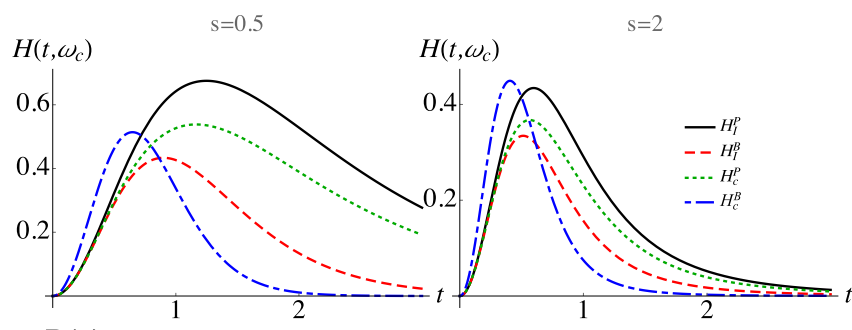

$R(s)$

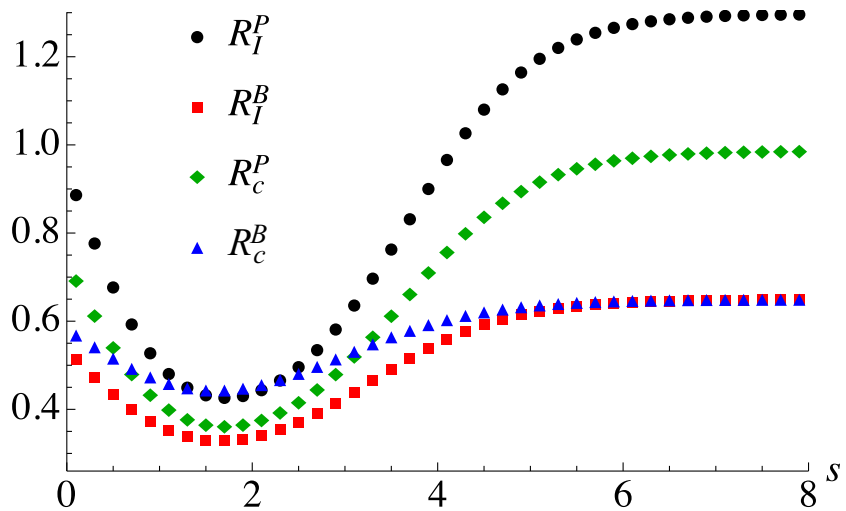

FIG. 4. Top: Fisher information as a function of time for two fixed values of the parameter $s$ in the cases of two qubits in independent environments in product (solid black line) and Bell (dashed red line) states and in a common environment prepared in a product (dotted green line) or Bell (dot-dashed blue line) state for $\omega_{c}=1$. Bottom: Comparison between the QSNR as a function of the Ohmicity $s$, obtained from the optimized quantum Fisher information, for four different initial conditions of the qubit: two qubits initially in a separable (black dots) or an entangled (red squares) state in independent reservoirs and two qubits in a common environment initialized in a product (green diamonds) or entangled (blue triangles) state.

values for $s$ are $s=\frac{1}{2}, s=1$, and $s=3$ [43], and they fall into this case, where a single qubit is the optimal probe. This is a relevant conclusion that tells us that the simplest quantum probe, a qubit, is sufficient to estimate the spectral parameter of the environment. However, we also found that there is a small range of the Ohmicity parameter where it is better to use two qubits prepared in a Bell state interacting with the same quantum bath in order to obtain a larger estimation precision.

In order to deepen our analysis to include states with a different amount of entanglement, we analyze the performances of Werner states $\rho_{W}$ as quantum probes, where $\rho_{W}=$ $p\left|\phi^{B}\right\rangle\left\langle\phi^{B}\right|+(1-p) \mathbb{I} / 4$ with $I$ the identity matrix and $\left|\phi^{B}\right\rangle$ one of the four Bell states. The parameter $p$ is related to the purity $P$ of the state through the relation $P=\left(1+3 p^{2}\right) / 4$ and the associated entanglement $E$ is nonzero only for $1 / 3<p<$ 1 and $E=(3 p-1) / 2$. The QFI for two qubits initialized in a Werner state and interacting with separate baths or a common environment takes the expression

$$
\begin{gathered}
H_{I}^{W}\left(t, \omega_{c}, p\right)=\frac{8 p^{2}(1+p)\left[\partial_{\omega_{c}} \Gamma\left(t, \omega_{c}\right)\right]^{2}}{(1+p)^{2} e^{4 \Gamma\left(t, \omega_{c}\right)}-4 p^{2}}, \\
H_{c}^{W}\left(t, \omega_{c}, p\right)=\frac{32 p^{2}(1+p)\left[\partial_{\omega_{c}} \Gamma\left(t, \omega_{c}\right)\right]^{2}}{(1+p)^{2} e^{8 \Gamma\left(t, \omega_{c}\right)}-4 p^{2}} .
\end{gathered}
$$

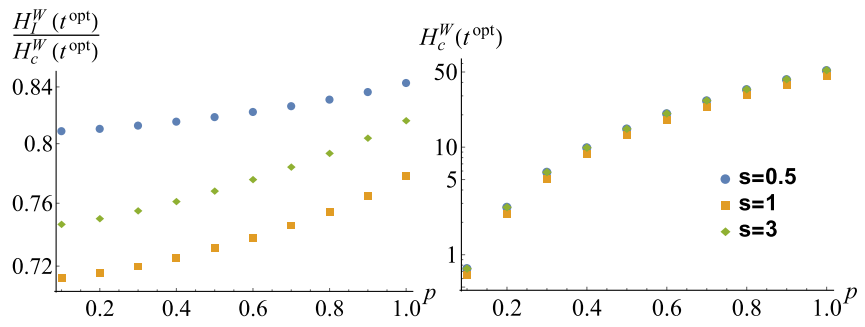

FIG. 5. Left: Ratio of the optimized QFI for Werner states in independent and common baths, as a function of the parameter $p$, for three different values of $s$. Right: Behavior of the optimal QFI for two qubits in a Werner state interacting in a common environment for $\omega_{c}=0.8$.

Figure 5 shows the ratio $\frac{H_{I}^{W}\left(t^{\mathrm{opt}}\right)}{H_{c}^{W}\left(t^{\mathrm{opt}}\right)}=\frac{R_{I}^{W}}{R_{c}^{W}}$ as a function of the parameter $p$ and for three different values of $s$. We first notice that, since the ratio is smaller than 1 , Werner states perform better in a common bath than in independent environments. Since Bell states $(p=1)$ permit one to achieve the largest precision, we can exclude the use of Werner states as optimal quantum probes and no improvement is gained in their use.

At last, we can ask ourselves what happens if we use $N$ qubits as a probe. As in the case of two qubits, the generalization to $N$ qubits cannot be done analytically, except for a few select cases. Here we extend the analysis of the QFI for Greenberger-Horne-Zeilinger (GHZ) states $\left|\psi_{\mathrm{GHZ}}\right\rangle=$ $\frac{1}{\sqrt{2}}(|000 \cdots\rangle+|111 \cdots\rangle)$ in independent and common baths, postponing a more complete discussion for future works. The QFI for the GHZ states reads

$$
\begin{aligned}
& H_{I}^{\mathrm{GHZ}}\left(t, \omega_{c}, N\right)=\frac{N^{2}\left[\partial_{\omega_{c}} \Gamma\left(t, \omega_{c}\right)\right]^{2}}{e^{2 N \Gamma\left(t, \omega_{c}\right)}-1}, \\
& H_{c}^{\mathrm{GHZ}}\left(t, \omega_{c}, N\right)=\frac{N^{4}\left[\partial_{\omega_{c}} \Gamma\left(t, \omega_{c}\right)\right]^{2}}{e^{2 N^{2} \Gamma\left(t, \omega_{c}\right)}-1} .
\end{aligned}
$$

The maximum of the QFI increases with the number of qubits $N$ and $H_{c}^{\mathrm{GHZ}}\left(t, \omega_{c}, N\right)$ is larger than $H_{I}^{\mathrm{GHZ}}\left(t, \omega_{c}, N\right)$ for fixed values of the parameters. However, these QFI remain smaller than the quantum Fisher information obtained using $N$ independent qubits as probes.

\section{CONCLUSIONS}

In this paper we have addressed the estimation of the cutoff frequency of an Ohmic reservoir using single-qubit and twoqubit quantum probes. The reservoir is made of an ensemble of noninteracting bosonic modes and the interaction between system and environment generates a dephasing map. We have evaluated the quantum Fisher information for different initial states of the probes, showing that, for a single-qubit probe, the optimal state preparation is the superposition $|+\rangle$, and that the optimal interacting time is inversely proportional to the cutoff frequency itself, $\omega_{c}$, such that the maximized QSNR is independent of the value of the cutoff frequency for any fixed value of the Ohmicity parameter $s$.

In order to understand if multiqubit quantum probes perform better than a single-qubit one, we also employed two noninteracting qubits to infer the value of $\omega_{c}$. Clearly, we 
can only compare specific initial states for the two-qubit case since we cannot provide the analytic expression for the two-qubit QFI for a generic initial state. For this reason we focused only on initial product and Bell states. In particular, we compare the precision, i.e., the QFI, obtained from four different scenarios, reported in Table I. We showed that also in these cases the QSNR does not depend on the value of $\omega_{c}$ and that for most values of $s$, including the most common cases $s=0.5,1$, and 3 [43], product states perform better than Bell states in estimating the cutoff frequency. This means that a single qubit is already optimal to infer the value of the cutoff frequency. However, we found that there exists a small range of parameters approximately $1.35<s<2.3$ where using a common environment scheme with two qubits initialized in a Bell state allows one to achieve a better estimation precision.

Our work paves the way for future developments, which include the estimation of the spectral parameters for an Ohmic reservoir at nonzero temperature and the study of system-bath couplings with different spectra.

\section{ACKNOWLEDGMENTS}

This work has been supported by EU through the collaborative H2020 project QuProCS (Grant No. 641277). F.S.S. thanks the QTLab group for the kind hospitality during her stay in Milan.
[1] H.-P. Breuer and F. Petruccione, The Theory of Open Quantum Systems (Oxford University Press, Oxford, UK, 2002).

[2] A. Abdelrahman et al., Nat. Commun. 8, 15712 (2017).

[3] T. J. Elliott and T. H. Johnson, Phys. Rev. A 93, 043612 (2016).

[4] M. Streif, A. Buchleitner, D. Jaksch, and J. Mur-Petit, Phys. Rev. A 94, 053634 (2016).

[5] F. Troiani and M. G. A. Paris, Phys. Rev. B 94, 115422 (2016).

[6] F. Cosco, M. Borrelli, F. Plastina, and S. Maniscalco, Phys. Rev. A 95, 053620 (2017).

[7] M. G. A. Paris, Int. J. Quantum Inf. 7, 125 (2009).

[8] C. Benedetti, F. Buscemi, P. Bordone, and M. G. A. Paris, Phys. Rev. A 89, 032114 (2014).

[9] C. Benedetti and M. G. A. Paris, Phys. Lett. A 378, 2495 (2014).

[10] A. Zwick, G. A. Alvarez, and G. Kurizki, Phys. Rev. Appl. 5, 014007 (2016).

[11] M. Bina, F. Grasselli, and M. G. A. Paris, Phys. Rev. A 97, 012125 (2018).

[12] A. Monras and M. G. A. Paris, Phys. Rev. Lett. 98, 160401 (2007).

[13] A. Fujiwara, Phys. Rev. A 63, 042304 (2001).

[14] A. Fujiwara and H. Imai, J. Phys. A: Math. Gen. 36, 8093 (2003).

[15] O. Pinel, P. Jian, N. Treps, C. Fabre, and D. Braun, Phys. Rev. A 88, 040102(R) (2013).

[16] G. Brida, I. P. Degiovanni, A. Florio, M. Genovese, P. Giorda, A. Meda, M. G. A. Paris, and A. Shurupov, Phys. Rev. Lett. 104, 100501 (2010).

[17] G. Brida, I. P. Degiovanni, A. Florio, M. Genovese, P. Giorda, A. Meda, M. G. A. Paris, and A. P. Shurupov, Phys. Rev. A 83, 052301 (2011).

[18] R. Blandino, M. G. Genoni, J. Etesse, M. Barbieri, M. G. A. Paris, P. Grangier, and R. Tualle-Brouri, Phys. Rev. Lett. 109, 180402 (2012).

[19] C. Benedetti, A. P Shurupov, M. G. A. Paris, G. Brida, and M. Genovese, Phys. Rev. A 87, 052136 (2013).

[20] M. G. Genoni, S. Olivares, and M. G. A. Paris, Phys. Rev. Lett 106, 153603 (2011).

[21] A. Monras, Phys. Rev. A 73, 033821 (2006).

[22] M. Bina, A. Allevi, M. Bondani, and S. Olivares, Sci. Rep. 6, 26025 (2016).

[23] M. Kacprowicz, R. Demkowicz-Dobrzanski, W. Wasilewski, K. Banaszek, and I. A. Walmsley, Nat. Photonics 4, 357 (2010).

[24] N. Spagnolo, C. Vitelli, V. G. Lucivero, V. Giovannetti, L. Maccone, and F. Sciarrino, Phys. Rev. Lett. 108, 233602 (2012).
[25] M. Brunelli, S. Olivares, and M. G. A. Paris, Phys. Rev. A 84, 032105 (2011).

[26] L. A. Correa, M. Mehboudi, G. Adesso, and A. Sanpera, Phys. Rev. Lett. 114, 220405 (2015).

[27] M. P. V. Stenberg, Y. R. Sanders, and F. K. Wilhelm, Phys. Rev. Lett. 113, 210404 (2014).

[28] M. Bina, I. Amelio, and M. G. A. Paris, Phys. Rev. E 93, 052118 (2016).

[29] M. A. C. Rossi, Matteo Bina, M. G. A. Paris, M. G. Genoni, G. Adesso, and T. Tufarelli, Quantum Sci. Technol. 2, 01LT01 (2017).

[30] D. Tamascelli, C. Benedetti, S. Olivares, and M. G. A. Paris, Phys. Rev. A 94, 042129 (2016).

[31] J. Nokkala, S. Maniscalco, and J. Piilo, arXiv:1708.09625.

[32] O. Astafiev, Yu. A. Pashkin, Y. Nakamura, T. Yamamoto, and J. S. Tsai, Phys. Rev. Lett. 96, 137001 (2006).

[33] J. D. Joannopoulos, R. D. Meade, and J. N. Winn, Photonic Crystals: Molding the Flow of Light (Princeton University Press, Princeton, NJ, 1995).

[34] C. Benedetti and M. G. A. Paris, Int. J. Quantum Inf. 12, 1461004 (2014).

[35] C. Addis, G. Brebner, P. Haikka, and S. Maniscalco, Phys. Rev. A 89, 024101 (2014).

[36] M. Palma, K.-A. Suominen, and A. K. Ekert, Proc. R. Soc. London A 452, 567 (1996).

[37] C. Addis, B. Bylicka, D. Chruściński, and S. Maniscalco, Phys. Rev. A 90, 052103 (2014).

[38] J. H. Reina, L. Quiroga, and N. F. Johnson, Phys. Rev. A 65, 032326 (2002).

[39] H.-S. Goan, C.-C. Jian, and P.-W. Chen, Phys. Rev. A 82, 012111 (2010).

[40] M. Ban, Phys. Lett. A 381, 2313 (2017).

[41] A. Lampo, J. Tuziemski, M. Lewenstein, and J. K. Korbicz, Phys. Rev. A 96, 012120 (2017).

[42] U. Weiss, Quantum Dissipative Systems (World Scientific, Singapore, 1999).

[43] J. Paavola, J. Piilo, K.-A. Suominen, and S. Maniscalco, Phys. Rev. A 79, 052120 (2009).

[44] P. M. V. B. Barone and A. O. Caldeira, Phys. Rev. A 43, 57 (1991).

[45] C. Benedetti, F. Buscemi, P. Bordone, and M. G. A. Paris, Phys. Rev. A 87, 052328 (2013). 